Revista Tecné, Episteme y Didaxis: TED. Año 2014, Número Extraordinario. ISSN Impreso: 0121-3814, ISSN web: 2323-0126

Memorias, Sexto Congreso Internacional sobre Formación de Profesores de Ciencias. 08 al 10 de octubre de 2014, Bogotá

\title{
Intervención didáctica basada en la postulación de un modelo de germinación a alcanzar con estudiantes universitarios: resultados preliminares
}

Olvera, Meztli', López-Mota, Ángel² y Tamayo, Óscar³.

Categoría 2. Trabajo de investigación (concluido)

\section{Resumen}

Se reporta que estudiantes de secundaria no comprenden la germinación debido a sus concepciones alternativas, las cuales están alejadas de la explicación científica; por ello, nuestro propósito fue diseñar, desarrollar, aplicar y evaluar una intervención didáctica basada en la modelización de la germinación con estudiantes universitarios, mediante el modelo científico escolar de arribo (MCEA). Para ello, construimos el MCEA de germinación y con este modelo como hipótesis, diseñamos la unidad didáctica que se aplicó. Ofrecemos resultados obtenidos de la primera etapa de aplicación, los cuales muestran que los modelos alcanzados sobre germinación de seis estudiantes de segundo semestre, contiene elementos, relaciones y condiciones propuestos en el MCEA; y el MCEA sirve para el análisis de los resultados de dicha etapa.

\section{Palabras clave}

Modelos, germinación, unidad didáctica

\section{Objetivo}

El objetivo general de este proyecto es valorar el logro del MCEA mediante una intervención didáctica basada en la modelización del fenómeno de germinación con estudiantes universitarios, utilizando el MCEA como referencia para el diseño de la unidad didáctica y como hipótesis a ser probada.

\section{Marco teórico}

Delimitación del problema

\footnotetext{
1 Universidad Pedagógica Nacional. meztli0101@hotmail.com

2 Universidad Pedagógica Nacional. alopezm@upn.mx

3 Universidad de Caldas, Universidad Autónoma de Manizales. oscar.tamayo@ucaldas.edu.co
} 
Revista Tecné, Episteme y Didaxis: TED. Año 2014, Número Extraordinario. ISSN Impreso: 0121-3814, ISSN web: 2323-0126

Memorias, Sexto Congreso Internacional sobre Formación de Profesores de Ciencias. 08 al 10 de octubre de 2014, Bogotá

Lin (2004) reporta que estudiantes de secundaria taiwaneses no comprenden la germinación debido a sus concepciones alternativas, tales como: 'Ias semillas provienen de la fotosíntesis', 'las semillas necesitan el agua y la luz solar para la fotosíntesis', las cuales están alejadas de la explicación científica acerca de este proceso. Así, hay un problema de aprendizaje que pretendemos solucionar con una propuesta de enseñanza sobre dicho fenómeno.

Los modelos y la modelización en Didáctica de las Ciencias

La modelización en la enseñanza de las ciencias tiene un fuerte fundamento epistemológico. Giere, entre otros autores en el campo de la epistemología, ha ideado marcos teóricos para concebir la naturaleza de la ciencia, y proporciona una plataforma conceptual para pensar la categoría de modelo en la enseñanza de las ciencias (Adúriz-Bravo e Izquierdo-Aymerich, 2009).

Giere (1999) establece que los modelos científicos son entidades abstractas idealizadas, definidas por sus afirmaciones correspondientes, y que su conexión con el mundo real se hace con afirmaciones (hipótesis teóricas) que aseveran similitud con éste; por ello, el núcleo de cualquier teoría científica no es un conjunto de afirmaciones (axiomas o leyes) sino un conjunto de modelos. Éstos pueden describirse mediante afirmaciones, pero no son únicamente un conjunto de afirmaciones.

Duschl (1997) coincide con Giere, al definir las teorías como las mejores creencias razonadas sobre el mundo que nos rodea: explicaciones -una síntesis de hechos, objetivos y métodos de la ciencia- que progresan con el tiempo y son validadas por una comunidad científica. Por lo anterior, ambos autores sugirieren que, bajo estas consideraciones sobre la naturaleza de hacer ciencia-modelizar-, es necesario cambiar la enseñanza de ésta.

\section{Ciencia escolar}

La ciencia escolar es considerada ciencia porque permite pensar mediante modelos, los cuales deben ser adecuados al mundo de los alumnos. Es, además, experimental y discursiva, proporciona autonomía, es autónoma, aplicada, diversa y rigurosa. Por lo que se considera que los alumnos que protagonicen la ciencia escolar, pueden dar sentido a sus aprendizajes $y$, en consecuencia, controlarlos y desarrollarlos con autonomía, adquiriendo competencias para poder aplicar sus conocimientos (Izquierdo, Espinet, García, Pujol y Sanmartí, 1999). 
Revista Tecné, Episteme y Didaxis: TED. Año 2014, Número Extraordinario. ISSN Impreso: 0121-3814, ISSN web: 2323-0126

Memorias, Sexto Congreso Internacional sobre Formación de Profesores de Ciencias. 08 al 10 de octubre de 2014, Bogotá

Modelización

En esta visión, enseñar ciencia implica favorecer la construcción de modelos científicos escolares por parte de los alumnos, con la intención de que les proporcionen representaciones y explicaciones de los hechos del mundo. Aprender implica que los alumnos deben pensar teóricamente a través de modelos y deben ser capaces de aplicarlos a la interpretación de hechos. Los modelos deben ser significativos para los alumnos y posibilitar su evolución a lo largo de la escolaridad y su vida (López-Mota, et. al. 2011).

Modelo Científico Escolar de Arribo (MCEA)

López-Mota y Rodríguez-Pineda (2013) retoman, a Izquierdo et al. (1999), Halloun (2004) y García y Sanmartí (2006) entre otros, en la necesidad de que los alumnos trasciendan de una visión memorística de hacer ciencia a una centrada en modelos y los procesos de modelización. Ello ayudará a los estudiantes a construir teorías y desarrollar habilidades cognitivas para construir conocimiento basado en experiencia. Con base en esta perspectiva, los autores postulan el "Modelo Científico Escolar de Arribo o MCEA".

El MCEA es una propuesta original para el diseño y evaluación de estrategias didácticas que busquen la construcción de modelos (mediante modelización) por parte de los estudiantes. El MCEA se propone como dispositivo teóricometodológico para acercar las ideas de los estudiantes a las científicas. La construcción del MCEA, supone homologar conceptualmente la manera de pensar de los estudiantes (ideas previas), el contenido de los programas de estudio y la concepción científica acerca de un fenómeno, para formular a dónde se quiere llegar en términos de modelos con la estrategia didáctica (ED) y evaluar el cambio de los estudiantes bajo los mismos términos (López-Mota y Rodríguez, 2013).

El MCEA, surge como una respuesta didáctica para los profesores que buscan cambiar la enseñanza y el aprendizaje de las ciencias tradicional, por otra basada en modelos y modelización a través del diseño de una ED sobre un fenómeno determinado.

El MCEA retoma la visión de modelo de Schwartz y colaboradores (2009): el modelo científico es una "representación abstracta y simplificada de un sistema que hace visibles sus rasgos clave y puede usarse para explicar y predecir fenómenos estudiados por la ciencia; el cual consiste de elementos, relaciones, operaciones y normas que rigen las interacciones" 
Revista Tecné, Episteme y Didaxis: TED. Año 2014, Número Extraordinario. ISSN Impreso: 0121-3814, ISSN web: 2323-0126

Memorias, Sexto Congreso Internacional sobre Formación de Profesores de Ciencias. 08 al 10 de octubre de 2014, Bogotá

EI MCEA implica la identificación de un fenómeno estudiado por la ciencia y lo mira desde el ámbito curricular, científico y de las ideas previas, en términos de modelos. Así, el MCEA es un dispositivo teórico-metodológico en el ámbito de la investigación didáctica de la ciencias, que permite orientar el diseño, la recolección de evidencias y su sistematización, así como la evaluación de una ED sustentada en los modelos y la modelización (López-Mota y Rodríguez-Pineda, 2013).

\section{Metodología}

Las acciones realizadas para conformar y poner a prueba el MCEA propuesto por López-Mota y Rodríguez-Pineda (2013) consistieron en:

- Construir un MCEA sobre germinación

- Inferir el modelo inicial de los estudiantes de germinación (MIE).

- Inferir el modelo curricular de germinación (MCU).

- Identificar el modelo científico de germinación (MC).

- Construir del MCEA mediante la tensión de los modelos anteriores

- Elaborar criterios de diseño de la unidad didáctica (UD)con fundamento en el MCEA sobre germinación: aspectos ontológicos y epistemológicos

- Diseñar la unidad didáctica de germinación

La UD consistió de tres etapas distribuidas a lo largo de un semestre (Tabla 1).

- Aplicar la unidad didáctica

La UD se aplicó a cinco estudiantes universitarios de segundo semestre de la carrera de Biología, en la Facultad de Estudios Superiores Iztacala (FES-I) de la Unam.

- Evaluar la estrategia didáctica

Los resultados obtenidos de la implementación de la UD, se evaluaron en relación con el MCEA y al modelo alcanzado por los estudiantes sobre germinación, como lo proponen López-Mota y Rodríguez (2013). 
Revista Tecné, Episteme y Didaxis: TED. Año 2014, Número Extraordinario. ISSN Impreso: 0121-3814, ISSN web: 2323-0126

Memorias, Sexto Congreso Internacional sobre Formación de Profesores de Ciencias. 08 al 10 de octubre de 2014, Bogotá

\section{Resultados}

Al inicio de la primera etapa de aplicación de la UD, obtuvimos información de las concepciones sobre germinación de cinco estudiantes de segundo semestre. Con base en ella, inferimos el modelo inicial de cada estudiante sobre germinación. Después de la aplicación de las actividades diseñadas en la primera etapa de la UD, obtuvimos nuevamente información de las concepciones de los estudiantes y se construyó el modelo alcanzado individual sobre germinación de los cinco estudiantes al final de la primera etapa (Tablas 2 a 6).

Al comparar los elementos de los modelos alcanzados de cada uno de los estudiantes con los elementos propuestos del MCEA (Tabla 7), se observa que de los siete elementos considerados en el MCEA, tres (semilla, agua y temperatura) se encuentran en cuatro de los cinco modelos alcanzados de los estudiantes. El oxígeno y la luz son otros elementos del MCEA que se encuentran presentes en los modelos alcanzados de los estudiantes. El oxígeno se encuentra en tres de los modelos alcanzados, y la luz solo se presenta en uno de los modelos. Sin embargo, al final de la primera etapa de aplicación de la estrategia didáctica, los elementos ATP y hormonas del MCEA no forman parte de ninguno de los modelos alcanzados por los estudiantes.

Los elementos semilla, agua y temperatura son necesarios para explicar el fenómeno de germinación; sin embargo, no son suficientes. Es necesario que los alumnos integren los demás elementos del MCEA, como el embrión y ATP, ya que sin ellos su modelo no podría explicar la emergencia de la radícula, evento considerado como el fin de la germinación.

En cuanto a las relaciones de los modelos alcanzados de los estudiantes sobre germinación (Tablas 3 a 7) en comparación con las relaciones del MCEA propuesto (Tabla1), se considera que ninguna de las relaciones del MCEA se incorporó a los modelos alcanzados de los estudiantes sobre germinación. Sin embargo, la relación de emergencia del MCEA se puede vislumbrar en cuatro de los cinco modelos alcanzados por los estudiantes (modelos B a E), pero bajo los términos: nacer, dar lugar, convertir y romper. La relación de emergencia, como se mencionó anteriormente, es crucial para explicar el fenómeno de germinación.

Con respecto a las condiciones propuestas en el MCEA sobre germinación, solamente imbibición (modelos C y E), reactivación del metabolismo (modelos A y E) y respiración (modelo E) están presentes en los modelos alcanzados de algunos 
Revista Tecné, Episteme y Didaxis: TED. Año 2014, Número Extraordinario. ISSN Impreso: 0121-3814, ISSN web: 2323-0126

Memorias, Sexto Congreso Internacional sobre Formación de Profesores de Ciencias. 08 al 10 de octubre de 2014, Bogotá

de los estudiantes. El hecho de considerar estas tres condiciones es trascendental, ya que como su índole lo indica, sin estas condiciones, el fenómeno de germinación no tendría lugar.

\section{Conclusiones}

Al final de la primera etapa de aplicación de la unidad didáctica y de la obtención de los resultados de dicha etapa, se puede concluir lo siguiente:

- El MCEA sirve de herramienta-guía para la elaboración y análisis de los resultados de la primera etapa de la unidad didáctica diseñada.

- Los alumnos cuentan con un modelo de germinación cercano al MCEA; sin embargo, requiere un modelo más cercano al MCEA para explicar la germinación.

\section{Referencias bibliográficas}

Adúriz-Bravo, A. \& Izquierdo-Aymerich, M. (2009). Un modelo de modelo científico para la enseñanza de las ciencias naturales. Revista Electrónica de Investigación en Educación en Ciencias, 4, pág. 40-48.

Duschl, R. (1997). Renovar la enseñanza de las Ciencias. Importancia de las Teorías y su desarrollo. Madrid, España: Narcea.

García, P. y Sanmartí, N. (2006). La modelización: una propuesta para repensar la ciencia que enseñamos. En Quintanilla y Adúriz-Bravo (Eds.), Enseñar Ciencias en el Nuevo Milenio, pág. 279-298. Ediciones Universidad Católica de Chile: Santiago de Chile.

Giere, R. N. (1999). Del realismo constructivo al realismo perspectivo. Enseñanza de las Ciencias, Número extra, pág. 9-13.

Izquierdo, M., Espinet, M., García, M.P., Pujol, R.M. \& Sanmartí, N. (1999). Caracterización y fundamentación de la ciencia escolar. Enseñanza de las Ciencias, Número extra, pág. 79-92.

Lin, S.-W. (2004). Development and application of a two-tier diagnostic test for high school students' understanding of flowering plant growth and development. International Journal of Science and Mathematics Education, 2, pág. 175199.

López-Mota, A., Rodríguez, D., Reyes, F., Flores, M., Martínez, T. \& López, C. (2011). Dos líneas de investigación para el diseño de estrategias didácticas en la 
Revista Tecné, Episteme y Didaxis: TED. Año 2014, Número Extraordinario. ISSN Impreso: 0121-3814, ISSN web: 2323-0126

Memorias, Sexto Congreso Internacional sobre Formación de Profesores de Ciencias. 08 al 10 de octubre de 2014, Bogotá

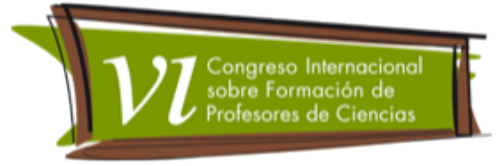

educación en ciencias: cambio conceptual y modelización. Balance. Ponencia presentada en el III Congreso Internacional y VIII Nacional de Investigación en Educación, Pedagogía y Formación Docente, Bogotá, Colombia, pág. 22- 24 , (paper).

López-Mota, A. \& Rodríguez-Pineda., D. (2013). Anclaje de los modelos y la modelización científica en estrategias didácticas. Enseñanza de las Ciencias, Número extra, 2008-2013.

Schwarz, C. V., Raiser, B. J., Davis, E. A., Kenyon, L., Achér, A., Fortus, D.,..., Krajcik, J. (2009). Developing a learning progression for scientific modeling: Making scientific modeling accessible and meaningful for learners. Journal of Research in Science Teaching, 46(6), pág. 632-654.

Tabla 1. Ełapas de la unidad didáctica diseña para la enseñanza y el aprendizaje de la germinación con estudiantes de segundo semestres de la carrera de Biología de la Facultad de Estudios Superiores Iztacala.

\begin{tabular}{|c|c|c|c|}
\hline ETAPA & PROPÓSITOS & $\begin{array}{l}\text { ASPECTOS DEL MCEA A } \\
\text { TRABAJAR }\end{array}$ & EVIDENCIAS \\
\hline \multirow[t]{3}{*}{1} & $\begin{array}{l}\text { - Conocer las concepciones alternativas } \\
\text { de los estudiantes sobre germinación. } \\
\text { - Explicitar el modelo de germinación de } \\
\text { los estudiantes. } \\
\text { - Poner a prueba el modelo de los } \\
\text { estudiantes. }\end{array}$ & $\begin{array}{l}\text { Expresión de los elementos, } \\
\text { relaciones y condiciones del } \\
\text { modelo inicial. }\end{array}$ & $\begin{array}{l}\text { Modelo de partida de los } \\
\text { estudiantes sobre } \\
\text { germinación de semillas }\end{array}$ \\
\hline & $\begin{array}{l}\text { - Diferenciar la fase de germinación del } \\
\text { ciclo de vida de las plantas. }\end{array}$ & $\begin{array}{l}\text { - Condiciones: dar fruto y } \\
\text { dar flor del modelo inicial. }\end{array}$ & \\
\hline & $\begin{array}{l}\text { - Reformulación del modelo inicial de los } \\
\text { estudiantes sobre germinación. } \\
\text { - Poner a prueba el modelo reformulado } \\
\text { de los estudiantes. }\end{array}$ & & $\begin{array}{l}\text { Modelo alcanzado de los } \\
\text { estudiantes } \\
\text { germinación }\end{array}$ \\
\hline \multirow[b]{2}{*}{2} & - Elaborar el protocolo de investigación. & $\begin{array}{l}\text { - Relación activación } \\
\text { - Condición reactivación del } \\
\text { metabolismo }\end{array}$ & $\begin{array}{l}\text { Protocolo de } \\
\text { investigación escrito sobre } \\
\text { germinación }\end{array}$ \\
\hline & $\begin{array}{l}\text { - Poner a prueba experimentalmente el } \\
\text { protocolo de investigación planteado } \\
\text { sobre germinación. }\end{array}$ & $\begin{array}{l}\text { - Condición de imbibición } \\
\text { - Relación de degradación } \\
\text { - Elemento enzimas }\end{array}$ & $\begin{array}{l}\text { Modelo alcanzado } 2 \text { de } \\
\text { los estudiantes sobre } \\
\text { germinación }\end{array}$ \\
\hline 3 & - Analizar y procesar los resultados. & & $\begin{array}{l}\text { Elaboración de tablas y } \\
\text { graficas que representen } \\
\text { los resultados. }\end{array}$ \\
\hline
\end{tabular}


Revista Tecné, Episteme y Didaxis: TED. Año 2014, Número Extraordinario. ISSN Impreso: 0121-3814, ISSN web: 2323-0126

Memorias, Sexto Congreso Internacional sobre Formación de Profesores de Ciencias. 08 al 10 de octubre de 2014, Bogotá

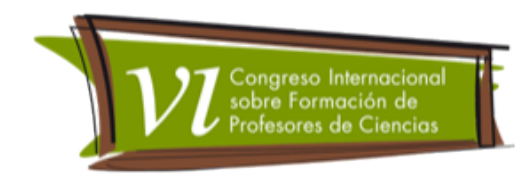

\begin{tabular}{|l|l|l|}
\hline $\begin{array}{l}\text { - Discutir los resultados } \\
\text { - Elaborar el reporte final del proyecto de } \\
\text { investigación. }\end{array}$ & Elemento hormonas & $\begin{array}{l}\text { - Reporte final escrito del } \\
\text { proyecto de } \\
\text { investigación. }\end{array}$ \\
\hline $\begin{array}{l}\text { - Plenaria } \\
\text { - Contrastación del modelo final con el } \\
\text { modelo inicial sobre germinación de los } \\
\text { estudiantes. }\end{array}$ & $\begin{array}{l}\text { - Modelo alcanzado final } \\
\text { de los estudiantes sobre } \\
\text { germinación }\end{array}$ \\
\hline
\end{tabular}

Tabla 2. Modelo alcanzado sobre el fenómeno de germinación de semillas del estudiante $A^{*}$ al final de la nrimera etana de la unidad didác.tica.

\begin{tabular}{|c|c|c|}
\hline Semilla & $\begin{array}{c}\text { Relaciones } \\
\text { Hinchar/Abrir } \\
\text { (semilla- } \\
\text { primeras hojas) }\end{array}$ & Condiciones \\
\hline Aguduración \\
\hline Temperatura & & $\begin{array}{c}\text { Crecimiento } \\
\text { metabolismo }\end{array}$ \\
\hline Oxígeno & & \\
\hline Bióxido de carbono & & \\
\hline Luz (si la requiere) & & \\
\hline
\end{tabular}

Tabla 3*.- Modelo alcanzado sobre el fenómeno de germinación de semillas del estudiante B* al final de la primera etapa de la unidad didáctica.

\begin{tabular}{|c|c|c|}
\hline Elementos & Relaciones & Condiciones \\
\hline $\begin{array}{l}\text { Semilla } \\
\text { Embrión }\end{array}$ & $\begin{array}{c}\text { Nacer } \\
\text { (nuevo vegetal- } \\
\text { planta madre) }\end{array}$ & Maduración \\
\hline Agua & & \\
\hline Oxígeno & & \\
\hline Temperatura & & \\
\hline Nuevo vegetal & & \\
\hline Planta madre & & \\
\hline
\end{tabular}


Revista Tecné, Episteme y Didaxis: TED. Año 2014, Número Extraordinario. ISSN Impreso: 0121-3814, ISSN web: 2323-0126

Memorias, Sexto Congreso Internacional sobre Formación de Profesores de Ciencias. 08 al 10 de octubre de 2014, Bogotá

Tabla 6*.- Modelo alcanzado sobre el fenómeno de germinación de semillas del estudiante E al final de la primera etapa de la unidad didáctica.

\begin{tabular}{|c|c|c|}
\hline $\begin{array}{c}\text { Elementos } \\
\text { (testa) }\end{array}$ & Relaciones & Condiciones \\
\hline $\begin{array}{c}\text { Embrión } \\
\text { (semilla- } \\
\text { plántula) }\end{array}$ & \\
\hline $\begin{array}{c}\text { Factores o } \\
\text { condiciones }\end{array}$ & & Imbibición \\
\hline Plántula & & \\
\hline
\end{tabular}

\begin{tabular}{|c|c|c|}
\hline Elementos & Relaciones & Condiciones \\
\hline $\begin{array}{c}\text { Semilla (enzimas) } \\
\text { Testa }\end{array}$ & $\begin{array}{c}\text { Convertir } \\
\text { (semilla-plántula) }\end{array}$ & $\begin{array}{c}\text {-Maduración morfológica } \\
\text { y fisiológica } \\
\text { - Viabilidad } \\
\text { - Dormición }\end{array}$ \\
\hline Ambiente/medio & Afectar/Interactuar & $\begin{array}{c}\text { Reactivación/aceleración } \\
\text { del metabolismo }\end{array}$ \\
\hline Agua & $\begin{array}{c}\text { Hinchar } \\
\text { (semilla-enzimas) }\end{array}$ & Imbibición \\
\hline Aireación & $\begin{array}{c}\text { Romper } \\
\text { (semilla-enzimas- } \\
\text { testa) }\end{array}$ & Respiración \\
\hline Temperatura & & Activación de enzimas \\
\hline $\begin{array}{c}\text { Plántula/planta } \\
\text { con primera hoja } \\
\text { real }\end{array}$ & & Crecimiento \\
\hline
\end{tabular}


Revista Tecné, Episteme y Didaxis: TED. Año 2014, Número Extraordinario. ISSN Impreso: 0121-3814, ISSN web: 2323-0126

Memorias, Sexto Congreso Internacional sobre Formación de Profesores de Ciencias. 08 al 10 de octubre de 2014, Bogotá

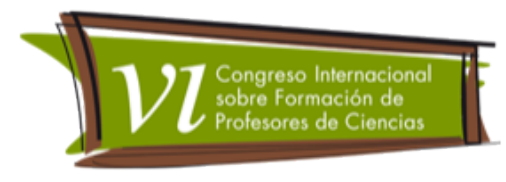

Tabla 7. Modelo Científico Escolar de Arribo (MCEA) para la enseñanza y el aprendizaje de la germinación de estudiantes de segundo semestres de la carrera de Biología de la Facultad de Estudios Superiores Iztacala.

\begin{tabular}{|c|c|c|}
\hline Elementos & Relaciones & Condiciones \\
\hline $\begin{array}{l}\text { Semilla (testa, hilio, micropilo: } \\
\text { región micropilar) } \\
\text { - Cotiledones o endospermo } \\
\text { (materiales de reserva: } \\
\text { carbohidratos, proteínas y } \\
\text { lípidos; y enzimas: alfa y beta } \\
\text { amilasas). } \\
\text { - Embrión (radícula, células) }\end{array}$ & $\begin{array}{c}\text { Absorción } \\
\text { (semilla: testa, hilio-agua) }\end{array}$ & $\begin{array}{l}\text { Madurez } \\
\text { Viabilidad } \\
\text { Latencia }\end{array}$ \\
\hline Agua & $\begin{array}{c}\text { Activación } \\
\text { (semilla: embrión-agua-luz solar- } \\
\text { temperatura-enzimas) }\end{array}$ & Imbibición \\
\hline Temperatura & & $\begin{array}{l}\text { Reactivación del } \\
\text { metabolismo }\end{array}$ \\
\hline Oxígeno & $\begin{array}{c}\text { Incremento } \\
\text { (semilla: embrión-oxígeno) }\end{array}$ & Respiración \\
\hline $\begin{array}{l}\text { Hormonas } \\
\text { - } \quad \text { Ácido absísico } \\
\text { - } \quad \text { Ácido giberélico }\end{array}$ & $\begin{array}{c}\text { Producción } \\
\text { (semilla: embrión-oxígeno-ATP) }\end{array}$ & Alargamiento celular \\
\hline \multirow[t]{3}{*}{ ATP (adenosin trifiosfato) } & $\begin{array}{c}\text { Alargamiento } \\
\text { (semilla: embrión-células) }\end{array}$ & Ruptura del endospermo \\
\hline & $\begin{array}{c}\text { Ablandamiento } \\
\text { (semilla: micrópilo: región } \\
\text { micropilar-hormonas) }\end{array}$ & Ruptura de la testa \\
\hline & $\begin{array}{c}\text { Emergencia } \\
\text { (semilla: embrión, radícula-testa) }\end{array}$ & Protrusión de la radícula \\
\hline
\end{tabular}

*Los modelos alcanzados inferidos corresponden a fragmentos identificados en las respuestas de cada uno de los estudiantes. Ejemplo: ¿Estás de acuerdo con la idea dos? SI ( ) NO(X). Las razones que apoyan mi elección son: No porque en Primera No Usa todos los elementos que Rodean a la Semilla para su Germinación, Solo los principales como lo es el Agua, Oxigeno y Temperatura. Agua, oxígeno y temperatura se consideraron elementos del modelo alcanzado del estudiante A. 